\title{
Síndrome de Burnout en Supervisores de Seguridad Industrial de Arequipa
}

\section{RESUMEN}

En este trabajo se exponen los resultados del estudio de las manifestaciones del síndrome de burnout en una muestra de 58 supervisores de seguridad industrial $(54.3 \%$ varones y $46.6 \%$ mujeres) con un rango de edad de 22 a 58 años. Se aplicó el Inventario de Burnout de Maslach forma general y se encontró que el $87 \%$ de los supervisores evaluados presentan niveles moderados de burnout, además, la baja realización personal tiene mayor grado de severidad de las tres dimensiones del síndrome. El tiempo de servicio se relaciona con el agotamiento emocional y la despersonalización, mientras que la edad se relaciona con la baja realización personal. Las mujeres se encuentran más afectadas por el síndrome, pero las diferencias con los varones no son significativas.

Palabras clave: estrés laboral, salud mental ocupacional, Síndrome de Burnout, supervisores

BURNout SYNDRome IN INDUSTRIAL SAFETy SUPERVISORS From AREQUiPa City

\section{ABSTRACT}

In this work, we expose the results of the study of burnout syndrome manifestations in a sample of 58 occupational safety supervisors $(54.3 \%$ male and $46.6 \%$ female) with an age range among 22 and 58 years old. The Maslach Burnout Inventory (General Survey) was applied and the results show that $87 \%$ percent of supervisors tested have moderate levels of burnout. Moreover, inefficacy reached highest levels of severity of three dimensions of burnout syndrome. Time of service was related to exhaustion and cynicism, meanwhile age was related to inefficacy. Women were more affected by the syndrome comparing to male, but these differences were not significant..

Keywords: Burnout syndrome, job stress, occupational mental health, supervisors

\section{INTRODUCCIÓN}

Las aproximaciones iniciales de Frederick Freudenberger y Cristina Maslach consideraban el síndrome de burnout como exclusivo de las profesiones de servicios humanos (PSH), debido a que quienes lo padecían eran primordialmente, profesionales que ayudan a otras personas, y que por la naturaleza de su trabajo están sometidos continuamente a demandas de alta implicación emocional, que se combinan con estresores organizacionales, afectando su salud mental y física a través de sentimientos de baja autoestima, despersonalización e ineficacia [1]. De ahí que se pensaba que las profesiones más vulnerables a este síndrome eran las de salud (medicina, enfermería, obstetricia, psicología, etc.), de seguridad (policías, vigilantes, etc.) y docencia (profesores de inicial, primaria, secundaria y enseñanza superior).

En ese sentido una primera aproximación que tuvimos a nivel local, nos permitió reportar que en los grupos profesionales que mencionamos, el síndrome de burnout tiene manifestaciones muy particulares. Por ejemplo, en una muestra de enfermeras, hallamos que el agotamiento emocional era la dimensión con niveles más severos y que la sobrecarga laboral y el número de hijos se relacionaban directamente con el síndrome. Para el caso de los policías, las mujeres exhibían niveles elevados de despersonalización y los varones de insatisfacción personal, además, el sueldo se relacionó negativamente con la ineficacia reportada por los varones de manera más fuerte que en las mujeres. Con los profesores que formaron parte de la muestra, el tipo de gestión educativa de la escuela permitía diferenciar dos perfiles: quienes trabajaban en escuelas públicas tenían niveles más altos de despersonalización, mientras que los que laboraban en colegios privados tenían más sentimientos de baja realización personal [2].

Sin embargo, hoy en día, el síndrome de burnout no se circunscribe solo a estas profesiones, pues ya hay varios estudios sobre burnout en deportistas [3], conductores [4], personal administrativo [5] y técnicos de seguridad laboral

\footnotetext{
* Psicólogo, Universidad Nacional de San Agustín y Maestro en CC. de la Educación con mención en Psicopedagogía cognitiva por la Universidad Nacional Pedro Ruíz Gallo. Diplomado en Gestión de recursos humanos y Sistemas integrados. Miembro del staff de profesionales del Centro de Medicina Ocupacional Integral (CEMOIN) y profesor investigador de la Universidad Católica San Pablo, donde dirige el Grupo de Investigación Psyché. E-mail: walterlizandro@hotmail.com

** Estudiante del quinto semestre del Programa Profesional de Psicología de la Universidad Católica San Pablo y miembro del Grupo de Investigación Psyché.
} 
[6], solo por mencionar algunos nuevos grupos profesionales que son objeto de estudio. También se han realizado diversas estandarizaciones de los instrumentos que evalúan el síndrome de burnout, en muestras multiocupacionales de Latinoamérica [7] [8] sin embargo, en la mayoría de nuestros países, no se considera todavía como una enfermedad ocupacional, a diferencia de España, donde desde 1995 ha sido incluida por su legislación laboral como una causa de deterioro físico y mental del trabajador [9]. En el Perú, aunque se cuenta con la Ley de Salud y Seguridad en el Trabajo $\mathrm{N}^{\circ}$ 29783 desde el año 2011, muy poca atención se ha prestado a la salud mental de los trabajadores.

El presente estudio se centra en las manifestaciones del síndrome de burnout en supervisores de seguridad del sector industrial de la ciudad de Arequipa, que tienen una diversidad de tareas y funciones, que bien pueden hacerles vulnerables a padecer este complejo sindrómico. Al respecto, el estudio de Gil-Monte, Núñez-Román y SelvaSantoyo con 312 técnicos de prevención de riesgos de España, reportó que los síntomas cardiovasculares y el agotamiento emocional fueron mayores en las mujeres, concluyendo que el síndrome de burnout parece ser una especie de mediador entre los la percepción de fuentes de estrés y la aparición de síntomas cardiovasculares [6]. En ese sentido, la sobrecarga laboral parece ser uno de los factores que más incide en la ocurrencia de este síndrome, y que además, los supervisores de seguridad laboral tienen índices elevados de sobrecarga laboral, por lo que sería necesario investigar la incidencia del síndrome de burnout en este grupo profesional y sus manifestaciones con respecto a las dimensiones de agotamiento emocional, despersonalización y baja realización personal.

Por ejemplo, en Chile, el 63\% de trabajadores de la Asociación Chilena de Seguridad reportó tener sobrecarga de trabajo [10], sin embargo las competencias que debe tener un gestor de la seguridad laboral, aún no se ha definido, muy a pesar de que en ese país, existe un sistema de salud ocupacional desde 1968 (Ley No 16744). Precisamente un estudio de Salazar et al., se dio a conocer recientemente, que el perfil de un gestor de seguridad debería abarcar conocimientos en análisis y gestión de riesgos, gestión de servicios y programas de higiene ocupacional, legislación y gestión empresarial, prevención y control de riesgos ocupacionales, promoción de la salud, epidemiología y estadística [11]. Sin embargo, más allá de los conocimientos que deba poseer el promotor o supervisor de seguridad, su principal función es la promoción de una cultura de seguridad entre los trabajadores [12], lo que supone habilidades de comunicación, enseñanza e investigación con la finalidad de ayudar a desarrollar estrategias de seguridad de gran alcance para sus organizaciones.

Por ello, es que el supervisor de seguridad debe tener un contacto cercano con los trabajadores, considerando que sus conductas tienen un efecto significativo en las actitudes que tienen los trabajadores hacia la seguridad laboral. En consecuencia, los supervisores son pieza clave para la seguridad por la influencia que tienen en los trabajadores, a través del contacto, su proximidad, el ejemplo que imparten y la autoridad que ejercen sobre ellos. En ese sentido, hay estudios que reportan que la supervisión es un buen predictor de la satisfacción laboral y la accidentabilidad de los trabajadores [13]. Lamentablemente, los trabajadores no acatan las normas de seguridad de manera diáfana y obediente, aún sabiendo los riesgos a los que se exponen. En un estudio en trabajadores del sector construcción encontramos que entre los motivos por los cuáles no usan los equipos de protección personal destacan la incomodidad y la falta de hábito [14].

A ese nivel debe trabajar el supervisor de seguridad con los trabajadores, indagando sus hábitos, sus actitudes, sus creencias, su personalidad, etc.; porque la salud y la seguridad están fuertemente ligadas a la manera en que las personas interpretan el trabajo y asignan sentido a lo ocurre en su jornada laboral [15]. Por ejemplo, la impulsividad como rasgo de la personalidad, se relaciona con mayor número de errores [16] y accidentes en el trabajo [17]. También se sabe que las personas con actitudes más negativas hacia la conducta de riesgo y mayores niveles de experiencia emocional tienen menor intención de repetirla, llevando a comportamientos más cautelosos y críticos [18]. Sin embargo la tendencia actual es que la seguridad en el trabajo dependa cada vez menos del control externo y que haya mayor autocontrol e involucramiento activo de parte del trabajador [19].

De esto se desprende, que la labor del supervisor de seguridad, el ingeniero de seguridad o el gestor de seguridad, debe desarrollar las capacitaciones ocupacionales sobre la base de la andragogía [20] y valerse de diversas técnicas de investigación como la observación, la psicometría, la sociometría, la entrevistas, el focus group, etc. para recoger datos de los trabajadores y valorar sus relaciones, causas e implicancias organizacionales y para la salud del trabajador [21]. Un importante apoyo para la labor del supervisor de seguridad es la psicología, que en los últimos años ha hecho un aporte significativo 
al mundo laboral, desde la prevención de riesgos ocupacionales, el clima de seguridad, conductas de riesgo, la ergonomía, etc. [22]; y las técnicas conductuales, cognitivas y socioculturales que se han desarrollado en torno a la seguridad en el trabajo [23].

De ahí que los gestores y supervisores de seguridad deban formarse también en seguridad basada en el comportamiento, ya que desde la época en que Heinrich inició los estudios de prevención de accidentes se sabe que el $88 \%$ de los accidentes se debe al factor humano [15], por ello, los trabajadores deben ser objeto de estudio y la principal motivación para mejorar los estándares de salud y seguridad, poniendo en marcha una cultura de seguridad que se base en los logros y el reconocimiento de los trabajadores, más que en estrategias punitivas [21]. Se deben tomar en cuenta también diversos factores organizacionales. El clima organizacional por ejemplo afecta directamente la seguridad de los trabajadores, de modo que un clima óptimo favorece la salud y reduce la frecuencia de accidentes [24]. El tamaño de la empresa también es otro factor a considerar, pues mientras más pequeñas son las empresas, menos se cumplen las normas de seguridad [25]. Los factores psicosociales del puesto, como las funciones laborales, las relaciones interpersonales, el diseño y contenido de las tareas, son también variables a tomar en cuenta para una eficiente gestión de la salud y la seguridad laboral [26]. Por ello, la seguridad laboral comienza con la asignación racional de las personas a su puesto de trabajo [27], pues el estrés en general y el síndrome de burnout en particular se generan en gran medida por un desbalance entre las demandas y las capacidades del individuo [26].

Pues bien, dadas las demandas laborales del supervisor de seguridad y la responsabilidad que asume para con la salud de los trabajadores, el contacto que tiene con ellos y los contextos organizacionales que limitan en muchos casos su trabajo, el lógico pensar que presenta niveles elevados de burnout y de sus tres dimensiones clásicas. El presente trabajo es un estudio descriptivo-correlacional que analiza las manifestaciones del agotamiento emocional, la despersonalización y la baja realización personal.

\section{MATERIALES Y MÉTODO}

La muestra está constituida por 58 personas que laboran asumiendo funciones de seguridad en sus respectivas empresas, ya sea como gestores de seguridad, supervisores, jefes de seguridad, etc. Del total de participantes $54.3 \%$ fueron varones y el $46.6 \%$ restante fueron mujeres. La edad promedio fue de 31 años con una desviación estándar de \pm 7.91 dentro de un rango de 22 a 58 años. El $73.7 \%$ de la muestra está casado y el $26.3 \%$ está soltero, $75.4 \%$ no tiene hijos y el $24.6 \%$ restante tiene entre uno y tres hijos. El $89.2 \%$ trabaja menos de cinco años en su empresa, el $2.75 \%$ entre cinco y diez años y el $8.1 \%$ trabaja más de diez años. En cuanto a los ingresos tenemos que el $4.4 \%$ gana menos del sueldo mínimo, $44.4 \%$ gana aproximadamente el mínimo y el $51.1 \%$ recibe una remuneración superior al sueldo mínimo. Para seleccionarlos se utilizó la técnica de grupos intactos, por tanto se trata de un muestreo no probabilístico.

Los trabajadores fueron evaluados en las instalaciones del Colegio de Ingenieros de Arequipa, dentro del marco de un curso de capacitación sobre la Ley de Salud y Seguridad en el Trabajo $N^{\circ} 29783$, justamente cuando se tocaba el tema de salud mental ocupacional. Se consideraron como válidos solo aquellos protocolos de respuesta que estuvieron completamente llenados y que pertenecían a supervisores de seguridad en sus respectivos puestos de trabajo. La aplicación de la prueba fue de manera colectiva y tomó aproximadamente 10 minutos. Durante el recojo de la información se resolvieron diversas inquietudes de los participantes y se les animó a llenar la prueba voluntaria y sinceramente y de forma anónima.

Se utilizó el Inventario de Burnout de Maslach, en su versión genérica. Este instrumento fue validado por Gil-Monte y Peiró [7] en una muestra multiocupacional de Colombia, y consta de 22 ítems en una escala tipo Likert que comprende respuesta desde "todos los días" hasta "nunca" con una valoración de 6 a 0 . El inventario se compone de las dimensiones de agotamiento emocional, despersonalización y baja realización personal. Para efectos de nuestro estudio determinamos el índice de consistencia interna a través del índice Alpha de Cronbach, y se obtuvo un valor de $\alpha=0.717$, lo que sugiere que brinda resultados confiables. Para recoger los datos sociolaborales se utilizó una ficha de datos personales.

\section{RESULTADOS}

Como se aprecia en el Gráfico 1, un 58.6\% tiene un nivel bajo de agotamiento emocional, $19 \%$ tiene un nivel moderado y $22.4 \%$ tiene un nivel alto. En cuanto a la despersonalización $51.7 \%$ tiene un nivel bajo, $24.1 \%$ moderado y el $24.1 \%$ restante también tiene un nivel alto. Además el $46.6 \%$ tiene baja realización personal en un nivel bajo, $22.4 \%$ en un nivel moderado y $31 \%$ en un nivel alto. Finalmente, solo el $5.2 \%$ tiene un nivel bajo del síndrome, el $87.9 \%$ tiene un nivel moderado y el $6.9 \%$ tiene un nivel alto de síndrome de Burnout. 
Gráfico 1. Porcentajes de los niveles de burnout y sus dimensiones en supervisores de seguridad

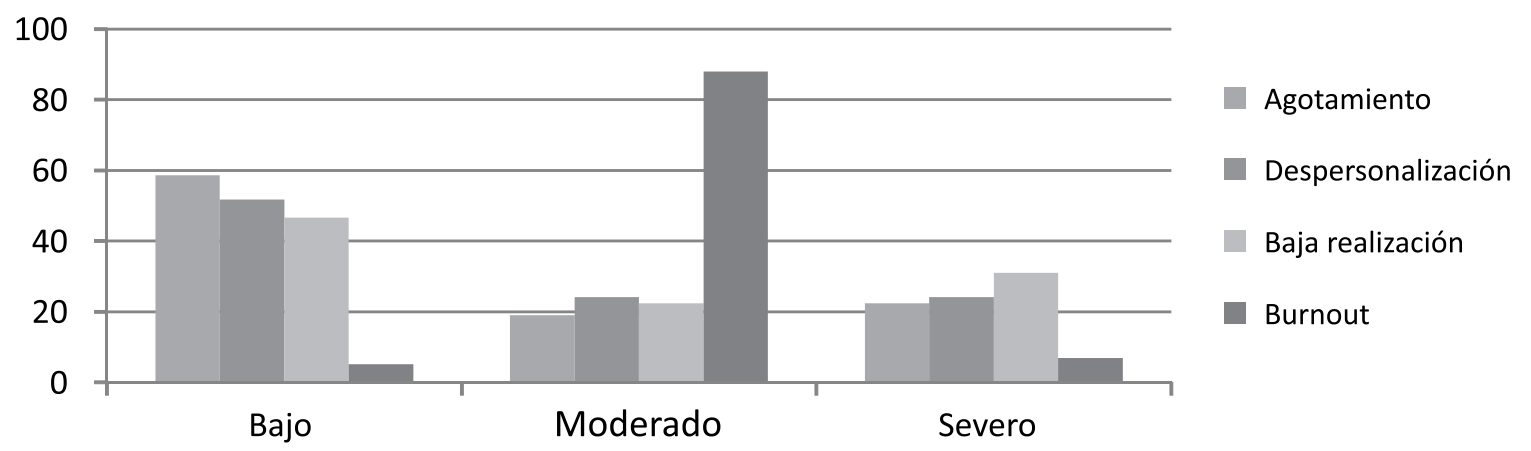

Se realizó también un análisis correlacional con la prueba de Pearson para las variables continuas y con la prueba Tau-b de Kendall para las variables ordinales. Los resultados se aprecian en la Tabla 1. Como se puede ver, el tiempo de trabajo se relaciona positivamente con la edad y el número de hijos, pero negativamente con el agotamiento emocional, la despersonalización y el síndrome de burnout. Los ingresos económicos se relacionan positivamente con el número de hijos y negativamente con el agotamiento emocional. La edad se relaciona positivamente con el número de hijos y la baja realización personal. Las tres dimensiones del síndrome de burnout se relacionan entre sí de manera positiva y significativa, siendo muy fuertes las relaciones entre el agotamiento emocional y la despersonalización, así como el agotamiento emocional, la despersonalización y el síndrome de burnout.
Por otro lado, para comparar los valores de las dimensiones del síndrome de burnout en función del sexo se hizo un análisis mediante la prueba $t$ student.

En la Tabla 2 se aprecian las medias obtenidas según el sexo en cada una de las dimensiones del síndrome de burnout, de modo que en agotamiento emocional, las mujeres registran valores más altos que los varones, en despersonalización son los varones quienes tienen medias más altas que las mujeres, en baja realización personal los puntajes son muy parejos, y en síndrome de burnout las mujeres tienen puntajes muy similares a los varones. Sin embargo, las diferencias entre varones y mujeres no son significativas, como se aprecia en la Tabla 3 donde se ha aplicado la prueba t student.

Tabla 1. Correlaciones entre variables

\begin{tabular}{lllllllll}
\hline & 1 & 2 & 3 & 4 & 5 & 6 & 7 & 8 \\
\hline Tiempo de trabajo & 1 & -0.129 & $0.429^{*}$ & $0.417^{*}$ & $-0.266^{*}$ & $-0.254^{*}$ & -0.010 & $-0.312^{*}$ \\
Ingresos & & 1 & 0.195 & $0.227^{*}$ & $-0.270^{*}$ & 0.024 & 0.165 & -0.095 \\
Edad & & & 1 & $0.594^{* *}$ & -0.195 & -0.102 & $0.264^{*}$ & -0.026 \\
Hijos & & & & 1 & -0.187 & -0.087 & 0.181 & -0.089 \\
Agotamiento & & & & & 1 & $0.454^{* *}$ & $-0.297^{*}$ & $0.764^{* *}$ \\
Despersonalización & & & & & & 1 & $-0.230^{*}$ & $0.625^{* *}$ \\
Baja Realización & & & & & & & 1 & $0.244^{*}$ \\
Síndrome de Burnout & & & & & & & & \\
\end{tabular}

${ }^{*} p<0.05 ;{ }^{* *} p<0.01$ 
Tabla 2. Medias del síndrome de burnout según el sexo

\begin{tabular}{lllll}
\hline \multirow{2}{*}{ Agotamiento } & \multicolumn{1}{c}{ Sexo } & N & Media & Desv. estándar \\
\hline \multirow{2}{*}{ Despersonalización } & varón & 31 & 17.580 & 9.793 \\
& mujer & 27 & 21.111 & 10.885 \\
\hline \multirow{2}{*}{ Baja realización } & varón & 31 & 7.258 & 5.489 \\
& mujer & 27 & 5.703 & 6.225 \\
\hline \multirow{2}{*}{ Síndrome de burnout } & varón & 31 & 36.612 & 8.822 \\
& mujer & 27 & 36.592 & 7.933 \\
\hline & varón & 31 & 61.354 & 12.862 \\
& mujer & 27 & 64.407 & 16.260 \\
\hline
\end{tabular}

Tabla 3. Comparación de medias con t student

\begin{tabular}{|c|c|c|c|c|c|c|c|c|c|}
\hline & \multicolumn{2}{|c|}{$\begin{array}{l}\text { Prueba de } \\
\text { Levene }\end{array}$} & \multicolumn{7}{|c|}{ Prueba t para equivalencia de medias } \\
\hline & \multirow{2}{*}{$\mathrm{F}$} & \multirow{2}{*}{ Sig. } & \multirow{2}{*}{$\mathrm{t}$} & \multirow{2}{*}{ df } & \multirow{2}{*}{$\begin{array}{l}\text { Sig. (2 } \\
\text { colas) }\end{array}$} & \multirow{2}{*}{$\begin{array}{l}\text { Diferencia } \\
\text { de medias }\end{array}$} & \multirow{2}{*}{$\begin{array}{c}\text { Error } \\
\text { estándar }\end{array}$} & \multicolumn{2}{|c|}{$95 \%$ de confianza } \\
\hline & & & & & & & & Inferior & Superior \\
\hline Agotamiento & ,475 & ,493 & $-1,300$ & 56 & ,199 & $-3,530$ & 2,715 & $-8,969$ & 1,908 \\
\hline Despersonalización & 226 & ,637 & 1,011 & 56 & ,317 & 1,554 & 1,537 & $-1,526$ & 4,635 \\
\hline Baja realización & 110 & ,741 & ,009 & 56 & ,993 & 020 & 2,216 & $-4,420$ & 4,461 \\
\hline Burnout & ,868 & ,355 &,- 798 & 56 & ,428 & $-3,052$ & 3,827 & $-10,719$ & 4,614 \\
\hline
\end{tabular}

Asimismo, hicimos comparaciones entre los trabajadores en función del sueldo que reciben a través de un análisis de varianza, y obtuvimos como resultado que no hay diferencias significativas entre las dimensiones del síndrome de burnout, sin embargo, al hacer el mismo procedimiento en función del tiempo de servicio, vimos que el análisis de varianza arroja diferencias significativas en el síndrome de burnout como valor total (ver Tabla 4).

Tabla 4. Análisis de varianza según el tiempo de servicio

\begin{tabular}{|c|c|c|c|c|c|c|c|c|}
\hline & & $\begin{array}{l}\text { Suma de } \\
\text { cuadrados }\end{array}$ & & df & $\begin{array}{r}\text { Cuadr } \\
\text { mec }\end{array}$ & $\begin{array}{l}\text { do de } \\
\text { as }\end{array}$ & $\mathrm{F}$ & Sig. \\
\hline \multirow{3}{*}{ Agotamiento } & Inter grupos & 451.931 & 2 & & 225.965 & & 2.165 & 0.130 \\
\hline & Intragrupos & 3547.636 & 34 & & 104.342 & & & \\
\hline & Total & 3999.567 & 36 & & & & & \\
\hline \multirow{3}{*}{ Despersonalización } & Inter grupos & 121.002 & 2 & & 60.501 & & 1.608 & 0.215 \\
\hline & Intragrupos & 1278.727 & 34 & & 37.609 & & & \\
\hline & Total & 1399.729 & 36 & & & & & \\
\hline \multirow{3}{*}{ Baja realización } & Inter grupos & 88.144 & & 2 & & 44.072 & 0.655 & 0.525 \\
\hline & Intragrupos & 2284.666 & & 34 & & 67.196 & & \\
\hline & Total & 2372.810 & & 36 & & & & \\
\hline \multirow{3}{*}{ Síndrome de Burnout } & Inter grupos & 1292.946 & & 2 & & 646.473 & 3.376 & 0.045 \\
\hline & Intragrupos & 6510.242 & & 34 & & 191.477 & & \\
\hline & Total & 7803.189 & & 36 & & & & \\
\hline
\end{tabular}


Finalmente, realizamos un análisis de regresión lineal para valorar cuál de las variables cuantitativas tienen mayor poder predictivo sobre el síndrome de burnout en los supervisores de seguridad. El modelo resultó significativo $(p<0.000)$ tomando como variables independientes el agotamiento emocional, la despersonalización, la baja realización personal, la edad y el número de hijos. Como se observa en la Tabla 5 , solo las dimensiones del síndrome de burnout tienen valor predictivo sobre el puntaje total.

Tabla 5. Coeficientes beta no estandarizados de las variables predictoras

\begin{tabular}{|c|c|c|c|c|c|}
\hline \multirow[t]{2}{*}{ Modelo } & \multicolumn{2}{|c|}{$\begin{array}{l}\text { Coeficientes no } \\
\text { estandarizados }\end{array}$} & \multirow{2}{*}{$\begin{array}{c}\text { Coef. estand. } \\
\text { Beta }\end{array}$} & \multirow[t]{2}{*}{$\mathrm{t}$} & \multirow[t]{2}{*}{ Sig. } \\
\hline & $B$ & E. típ. & & & \\
\hline (Constante) & 0.413 & 2.644 & & 0.156 & 0.876 \\
\hline Agotamiento & 1.030 & 0.043 & 0.759 & 23.885 & 0.000 \\
\hline Despersonalización & 1.034 & 0.073 & 0.431 & 14.169 & 0.000 \\
\hline Baja realización & 0.963 & 0.050 & 0.558 & 19.208 & 0.000 \\
\hline Edad & 0.025 & 0.050 & 0.017 & 0.510 & 0.612 \\
\hline Número de hijos & -0.340 & 0.535 & -0.021 & -0.637 & 0.527 \\
\hline
\end{tabular}

\section{DISCUSIÓN}

La psicología organizacional se ha desarrollado mucho durante el siglo XX, en parte por el impulso que le ha dado la investigación bélica donde se aplicaron profusamente técnicas de selección de personal para reclutar soladados [28]. Por otro lado, el desarrollo del mercado laboral, los avances tecnológicos y las economías globales; han diversificado las labores tradicionales de esta rama de la psicología, generando el surgimiento de nuevas especialidades [23]. Una de las especialidades que ha tenido un crecimiento muy significativo es la psicología de la seguridad y la salud ocupacional, que guarda íntima relación con la higiene ocupacional, la ergonomía y la ingeniería conductual [29].

Desde esta rama se investigan las fuentes clásicas de las enfermedades ocupacionales como la exposición al polvo [30], o el uso de equipos de protección personal [14], pero con un enfoque psicológico. Esto quiere decir que se analizan las motivaciones, conductas y creencias de los trabajadores como fenómenos mediadores de la salud y la seguridad en el trabajo. Aunque en un primer momento la psicología aplicada a la seguridad buscaba detectar las características individuales de riesgo de los trabajadores, hoy se orienta además, al estudio de las conductas que favorecen la gestión de riesgos laborales [17].

Uno de los fenómenos en los que se ha enfocado la psicología de la salud ocupacional es el estrés laboral, que puede entenderse como un estado de tensión laboral ocasionado por estímulos relacionados con el trabajo que tienen una presencia cotidiana. Por ejemplo en un estudio previo con personal de una empresa que comercializa y distribuye equipos de seguridad, encontramos que los estresores que se reportaban con mayor frecuencia eran los que se relacionaban con los hábitos de sueño y alimentación [31]. Por otro lado, aunque el estrés laboral tiene un origen en el trabajo, no quiere decir que los roles familiares carezcan de injerencia en los estados emocionales del trabajador. Sin embargo, es solo recientemente que se han analizado las relaciones entre la familia y el trabajo, concluyéndose que hay una correlación negativa entre los conflictos trabajo-familia y el bienestar del trabajador [27]. Por ejemplo, en un estudio con una amplia muestra de mineros en el Perú, Salvatierra encontró que los estilos de vida de los trabajadores en su núcleo familiar afectaban su desempeño laboral así como su salud y seguridad en el trabajo [12].

Es importante considerar que la cantidad de trabajadores afectado por estrés laboral ha aumentado de forma exponencial. El año 2005 la Organización Internacional de Trabajo (OIT) reportó 1,1 millones de enfermedades y accidentes de trabajo, siendo el estrés crónico la causa principal [26]. Para el 2010 la OIT amplió su lista de enfermedades profesionales a 116, lo que hace suponer que el estrés crónico (burnout) pronto será considerado como una enfermedad profesional, aunque en países como España, el síndrome de burnout ya es considerado como una enfermedad profesional9. Precisamente, en dicho país, un estudio señaló que en 44 de 85 demandas laborales, 
se estimó acreditada la presencia del síndrome de burnout [1]. Este síndrome es una reacción afectiva a un estrés prolongado en el contexto laboral, cuyo síntoma patognomónico es el agotamiento paulatino de los recursos energéticos intrínsecos de la persona, e incluye fatiga, cansancio cognitivo y agotamiento emocional8. De hecho, aunque también son signos de presencia de burnout, la despersonalización y la baja realización personal, el agotamiento emocional es el síntoma que más se relaciona y que mejor predice la ocurrencia de este síndrome [2].

En el presente estudio evaluamos y analizamos las manifestaciones del síndrome de burnout en una muestra de supervisores de seguridad que laboran en la ciudad de Arequipa. Los resultados que encontramos indican que el síntoma que presenta mayor incidencia es la baja realización personal, y que un pequeño porcentaje tenía manifestaciones severas del síndrome (6.9\%). Sin embargo, aproximadamente, el $87 \%$ de la muestra tenía índices moderados del síndrome de burnout, lo que podría ser un indicador de alarma en este grupo ocupacional. Entre las razones por las que los gestores de la seguridad podrían tener este síndrome, se enlistan la cultura organizacional [15], la sobrecarga laboral [26] y disposiciones personales [32]. En el primer caso, la mayoría de empresas en el Perú no cuenta con las estructuras ni la organización pertinente para promover la salud mental en el trabajo. En ese sentido, contar con un psicólogo en el trabajo sería de mucha utilidad para manejar el estrés laboral [33]. Por otro lado, dado que la principal función de los supervisores o gestores de seguridad es la promoción de la salud y ello implica llevar a cabo una gran diversidad de tareas y actividades, es lógico pensar que presentan sobrecarga laboral. Aunque no hemos valorado la sobre carga laboral a través de las horas dedicadas al trabajo o la cantidad de funciones que realizan los supervisores, es una explicación muy plausible considerando las características intrínsecas de las responsabilidades que desempeñan.

En ese sentido, encontramos que las mujeres se encuentran más afectadas por el síndrome de burnout, pero las diferencias no resultaron significativas. Entre los factores que explican estas diferencias se tiene la desigualdad socioeconómica, la falta de apoyo social a la mujer, el trabajo en exceso, la falta de autonomía y la carga familiar [26]. También encontramos que los trabajadores que tienen menos tiempo de servicio son los que presentan niveles más elevados del síndrome, hecho que ha sido confirmado por diversos estudios, donde se aprecia que los trabajadores con menos de cuatro años y los que tienen más de veinte años de trabajo son más propensos a padecer burnout [32]. En el primer caso, la inexperiencia sería un factor gatillador mientras que en los trabajadores con mayor tiempo de servicio, el agotamiento es el factor desencadenante. Asimismo, en los trabajadores jóvenes, puede verse una falta de especialización, pues en el Perú solo existen dos universidades a nivel nacional donde se forma de manera profesional a ingenieros de seguridad, siendo la mayoría de supervisores, profesionales de otras carreras como ingeniería industrial, mecánica, eléctrica o con formación técnica de mecánica de producción, etc. De hecho, en la presente investigación los trabajadores de menor edad son los que presentan más baja realización personal.

A esto se suma que los profesionales de la salud y la seguridad industrial no se focalizan en cuestiones como la seguridad basada en el comportamiento o la psicología de la salud ocupacional, sino más bien, se centran en los factores ambientales, los equipos de protección, etc. Es decir, que el factor psicológico es considerado como secundario, cuando en realidad existen relaciones bidireccionales entre ambos factores, de manera que así como las condiciones de trabajo pueden ser fuente de desequilibrio que genera estrés, problemas de salud y baja productividad; las creencias, hábitos y la personalidad de los trabajadores, impactan negativamente en el trabajo a través del clima laboral, la accidentabilidad y la motivación, produciéndose un círculo vicioso [27], que es responsabilidad del supervisor de seguridad. Con esto no queremos decir que la responsabilidad es exclusiva del supervisor, pues cada trabajador y el empleador tienen cierto grado de responsabilidad sobre la salud y la seguridad laboral, pero es el gestor de la seguridad, quien tiene como rol la minimización o neutralización de los diversos factores potencialmente nocivos para la salud física o mental en su organización, para lo cual debe convocar a todos los miembros de la comunidad laboral.

Por ello consideramos que los supervisores de seguridad deberían estar mejor entrenados para atender la salud mental de los trabajadores, a través de la implementación de técnicas de tamizaje, la organización de capacitaciones sobre estrés laboral, síndrome de burnout y técnicas de afrontamiento; pues muchos trabajadores optan por maneras poco saludables para hacer frente al estrés como el consumo de alcohol34. Además, serían muy útiles técnicas de trabajo colectivo como la ergonomía participativa [35], o las metodologías psicoeducativas familiares [12]. 
En suma la labor del supervisor de seguridad como principal promotor de la salud y la seguridad laboral, presenta riesgos psicosociales de alto impacto para su salud mental, y el síndrome de burnout es solo uno de varios factores. Es necesario destacar la importancia de una formación integral para este grupo profesional, y la correcta selección de profesionales especializados en este tipo de puestos dentro de las empresas de diversos rubros productivos, pero especialmente donde hay más casos de siniestralidad laboral, como son la industria, el sector construcción y la minería.

Esperamos que este estudio que forma parte de una línea de investigación sobre el síndrome de burnout que se desarrolla en nuestra localidad, permita concientizar a las autoridades, los empresarios y los profesionales de la salud, sobre la importancia de la salud ocupacional y la necesidad de incorporar cada vez más la prevención de riesgos psicosociales en el trabajo como el estrés crónico.

\section{REFERENCIAS}

[1] Tejero R, González D, Delgado S. El burnout como forma de estrés laboral y su dimensión forense. Rev. Cub. Salud Trab. 2013; 14(1): 5166.

[2] Arias WL, Jiménez, NA. Estrés crónico en el trabajo: Estudios sobre el síndrome de burnout en Arequipa. Arequipa: Universidad Católica San Pablo; 2012.

[3] Martín M, Campos A, Jiménez-Beatty JE, Martínez J. Calidad de vida y estés laboral: la incidencia del burnout en el deporte de alto rendimiento madrileño. Rev. int. cienc. deporte. 2007; 3(3): 62-77.

[4] Arias WL, Mendoza del Solar L, Masías MA. Síndrome de burnout en conductores de transporte público de la ciudad de Arequipa. Rev. Per. Psi. Trab. Soc. 2013; 2(2): 111-122.

[5] Arias WL, Riveros P, Salas XS. Espiritualidad en el ambiente laboral, estrés crónico (burnout) y estilos de afrontamiento en trabajadores de una empresa de servicios educativos. Cienc Trab. 2012; 14(44): 195-200.

[6] Gil-Monte PR, Núñez-Román EM, SelvaSantoyo Y. Relación entre el síndrome de quemarse por el trabajo (burnout) y síntomas cardiovasculares: Un estudio en técnicos de prevención de riesgos laborales. Inter. J. Psychol. 2006; 40(2): 227-232.
[7] Gil-Monte PR, Peiró JM. Validez factorial del Maslach Burnout Inventory en una muestra multiocupacional. Psicothema. 1999; 11(3): 679-689.

[8] Millán AC, D’Auberette ME. Propiedades psicométricas del Maslach Burnout InventoryGS en una muestra multiocupacional venezolana. Rev. Psicol. (Lima). 2012; 30(1): 103-128.

[9] Gil-Monte PR. El síndrome de quemarse por el trabajo (burnout). Madrid: Pirámide; 2005.

[10] Flores R, Bastías M. Determinación de enfermedad profesional y estudio de puesto de trabajo. Cienc Trab. 2011; 13(39): 36-43.

[11] Salazar AM, Frenz P, Valdivia L, Hurtado I. Evaluación de competencias de los gestores de la salud y seguridad ocupacional en Chile. Cienc Trab. 2013; 15(48): 114-123.

[12] Salvatierra B. Cultura de seguridad laboral para el minero. Lima: Noceda: 2011.

[13] Meliá JL, Sesé A. Supervisor's safety response: a multisample confirmatory factor analysis. Psicothema. 2007; 19(2): 231-238.

[14] Arias WL. Uso y desuso de equipos de protección personal en trabajadores de construcción. Cienc Trab. 2011; 13(40): 119-124.

[15] Finkelstein R, Salas F. Prevención de riesgos desde el observador: Un paradigma cultural. Cienc Trab. 2011; 13(39): 44-52.

[16] Buela-Casal G, Carretero-Dios H, De los Santos-Roig M. La reflexividad-impulsividad como una dimensión continua: validación del sistema de clasificación de Salkind y Wright (1977). Rev. Latinoam. Psicol. 2001; 33(2): 149-157.

[17] Sannino D, Gallardo M, Ackernecht C, López-Mena L. Evaluación conductual de la impulsividad aplicada al control de la accidentabilidad. Cienc Trab. 2012; 14(43): 105-111.

[18] Carrera P, Caballero A, Sánchez F, Blanco A. Emociones mixtas y conductas de riesgo. Rev. Latinoam. Psicol. 2005; 37(1): 119-130.

[19] López-Mena L. Habilidades de autocontrol aplicadas a la seguridad en el trabajo. Rev. Psicol. (Chile). 2000; 9: 1-17.

[20] Ackernecht C. Relación entre la edad y accidentabilidad en trabajadores del sector forestal. Cienc Trab. 2010; 12(38): 414-422. 
[21] Cabeza MA. Sistemas de incentivos basados en el reconocimiento. Un enfoque más estratégico. Rev. Venezol. Ana. Coyuntura. 2002; 8(1): 201-208.

[22] Marán D, Soro G, Zanotta T. La seguridad, ¿se aprende? Psicología y formación sobre seguridad en los lugares de trabajo. Rev. Psicodidáctica. 2007; 12(1): 107-120.

[23] Arias WL. Aportes de la psicología a la seguridad industrial y la salud ocupacional. Rev. psicol. Arequipa. 2011; 1(2): 134-145.

[24] Arias WL, Zegarra J. Clima organizacional, síndrome de burnout y afrontamiento en trabajadores de un hospital oncológico de Arequipa. Rev. psicol. (Trujillo). 2013; 15(1): 37-54.

[25] Salazar JG, Aranda C, Pando M, Gómez G, González R. Seguridad, salud y percepción de factores personales y organizacionales en la industria manufacturera. Cienc Trab. 2010; 12(38): 397-402.

[26] Fernández D, Ramírez JA. Síndrome de quemarse por el trabajo en mujeres trabajadoras de la industria petrolera en México. Rev. Cub. Salud Trab. 2013; 14(2): 3-11.

[27] Guerrero J, Puerto YI. Productividad, trabajo y salud: la perspectiva psicosocial. Rev. Colom. Psicol. 2007; 16: 203-234.

[28] Carpintero H. La International Association of Applied Psychology. Participación española en sus congresos (1920-1958). Rev. Hist. Psicol. 2000; 21(2-3): 415-432.

[29] Arias WL. Revisión histórica de la salud ocupacional y la seguridad industrial. Rev. Cub. Salud. Trab. 2012; 13(3): 54-52.

[30] Gómez-Yépez ME, Cremades LV. Análisis de la incidencia de patologías respiratorias por exposición al polvo de madera en los carpinteros de Quindío (Colombia). Cienc Trab. 2010; 12(38): 433-439.

[31] Arias WL. Estrés laboral en trabajadores desde el enfoque de los sucesos vitales. Rev. Cub. Salud Pub. 2012; 38(4): 525-535.

[32] Gil-Monte PR, Peiró JM. Perspectivas teóricas y modelos interpretativos para el estudio del síndrome de quemarse por el trabajo. A. psicol. 1999; 15(2): 261-268.

[33] Contreras CA. Estilos de vida promotores de la salud en trabajadores de una plataforma marina en el Golfo de México. Cienc Trab. 2011; 13(41): 181-186.

[34] Arias WL. Estrés laboral y consumo de sustancias psicoactivas (SPA) desde un enfoque de la salud ocupacional. Rev. Per. Psi. Trab. Soc. 2012; 1(1): 107-118.

[35] León MY. Ergonomía participativa en cajeras de una gran tienda. Cienc Trab. 2011; 13(39): 57-63. 